\title{
Nodal management and upstaging of disease: initial results from the Italian VATS Lobectomy Registry
}

\author{
Alessandro Bertani ${ }^{1}$, Alessandro Gonfiotti ${ }^{2}$, Mario Nosotti ${ }^{3}$, Paolo Albino Ferrari ${ }^{1}$, Lavinia De \\ Monte $^{1}$, Emanuele Russo ${ }^{1}$, Gioacchino Di Paola ${ }^{1}$, Piero Solli ${ }^{4}$, Andrea Droghetti ${ }^{5}$, Luca Bertolaccini ${ }^{4}$, \\ Roberto Crisci $^{6}$; Italian VATS Group ${ }^{7 *}$
}

${ }^{1}$ Division of Thoracic Surgery and Lung Transplantation, Department for the Treatment and Study of Cardiothoracic Diseases and Cardiothoracic Transplantation, IRCCS ISMETT, Palermo, Italy; ${ }^{2}$ Division of Thoracic Surgery, Careggi Hospital, Firenze, Italy; ${ }^{3}$ Division of Thoracic Surgery, Policlinico Ca'Granda, Milano, Italy; ${ }^{4}$ Department of Thoracic Surgery, AUSL Romagna Teaching Hospital, Forlì, Italy; ${ }^{5}$ Division of Thoracic Surgery, ASST Mantova-Cremona, Mantova, Italy; ${ }^{6}$ Division of Thoracic Surgery, Università dell'Aquila, L'Aquila, Italy; ${ }^{7}$ Collaborators of the Italian VATS Group

Contributions: (I) Conception and design: A Bertani, A Gonfiotti, M Nosotti, R Crisci; (II) Administrative support: A Bertani, G Di Paola, P Solli, A Droghetti, L Bertolaccini; (III) Provision of study materials or patients: All authors and collaborators; (IV) Collection and assembly of data: A Bertani, A Gonfiotti, M Nosotti, PA Ferrari, L De Monte, E Russo, G Di Paola; (V) Data analysis and interpretation: A Bertani, A Gonfiotti, M Nosotti, G Di Paola, L Bertolaccini; (VI) Manuscript writing: All authors; (VII) Final approval of manuscript: All authors.

Correspondence to: Alessandro Bertani. Division of Thoracic Surgery and Lung Transplantation, Department for the Treatment and Study of Cardiothoracic Diseases and Cardiothoracic Transplantation, IRCCS ISMETT, Via Tricomi 5, 90127, Palermo, Italy. Email: abertani@ismett.edu.

Background: VATS lobectomy is an established option for the treatment of early-stage NSCLC. Complete lymph node dissection (CD), systematic sampling (SS) or resecting a specific number of lymph nodes (LNs) and stations are possible intra-operative LN management strategies.

Methods: All VATS lobectomies from the "Italian VATS Group" prospective database were retrospectively reviewed. The type of surgical approach (CD or SS), number of $\mathrm{LN}$ resected (RN), the positive/resected LN ratio (LNR) and the number and types of positive LN stations were recorded. The rates of nodal upstaging were assessed based on different LN management strategies.

Results: CD was the most frequent approach (72.3\%). Nodal upstaging rates were 6.03\% (N0-to-N1), $5.45 \%$ (N0-to-N2), and $0.58 \%$ (N1-to-N2). There was no difference in N1 or N2 upstaging rates between $\mathrm{CD}$ and SS. The number of resected nodes was correlated with both N1 (OR =1.02; CI, 1.01-1.04; P=0.03) and N2 (OR =1.02; CI, 1.01-1.05; $\mathrm{P}=0.001)$ upstaging. Resecting 12 nodes had the best ability to predict upstaging ( $6 \mathrm{~N} 1 \mathrm{LN}$ or $7 \mathrm{~N} 2 \mathrm{LN})$. The finding of two positive LN stations best predicted N2 upstaging [area under the curve (AUC) of receiver operating characteristic $(\mathrm{ROC})=0.98]$.

Conclusions: Nodal upstaging (and, indirectly, the effectiveness of intra-operative nodal management) cannot be predicted based on the surgical technique (CD or SS). A quantitative assessment of intra-operative LN management may be a more appropriate and measurable approach to justify the extension of LN resection during VATS lobectomy.

Keywords: VATS lobectomy; lymph node (LN); nodal upstaging

Submitted May 03, 2017. Accepted for publication May 16, 2017.

doi: $10.21037 /$ jtd.2017.06.12

View this article at: http://dx.doi.org/10.21037/jtd.2017.06.12 


\section{Introduction}

VATS lobectomy has recently gained acceptance as the ideal treatment for early stage non-small cell lung cancer (NSCLC) (1). The short- and long-term results are comparable or superior to those of open pulmonary lobectomy. Patients' benefits include less pain, an earlier hospital discharge, and an improved ability to tolerate adjuvant treatments $(2,3)$.

The overall and disease-free survival of patients undergoing lung resection is related to the final pathological nodal stage of disease. Provided an appropriate intraoperative lymph node ( $\mathrm{LN}$ ) management, the absence of nodal metastases correlates with improved survival $(4,5)$.

Different LN resection strategies have been suggested. Complete dissection of all hilar and mediastinal lymphnodes (CD) and systematic sampling (SS) of predeterminate nodal stations (SS) are the two most frequent approaches. CD refers to the removal of all the lymphatic and fatty tissue within specific anatomic landmarks; SS refers to the retrieval of one or more sample nodes from each identified station (6).

The interpretation of the role of both CD and SS may present a significant bias, considering that both techniques vary on the surgeon's practice and are very difficult to define quantitatively. Therefore, it is difficult to measure the risk and benefits of one or the other strategy.

Other approaches for the evaluation of $\mathrm{LN}$ management during lung resection include the number of resected $\mathrm{LN}$, the $\mathrm{LN}$ ratio (LNR), the number of resected $\mathrm{LN}$ stations, and nodal upstaging (7-11).

Nodal upstaging, defined as the finding of a higher $\mathrm{N}$ value on the final pathologic specimen compared to the preoperative clinical $\mathrm{N}$, has been identified as a viable predictor of the adequacy of nodal management and, indirectly, as a surrogate for the identification of patients who are at risk of survival (12).

The possibility to perform an adequate $\mathrm{LN}$ resection during VATS lobectomy compared to open lobectomy has been extensively demonstrated (13-15).

Large, prospective, multi-institutional VATS lobectomy series from cancer databases may lack adequate information on intra-operative nodal management. More consistent and detailed data on intra-operative $\mathrm{LN}$ management may be available from retrospective, single center experiences, with the limitation of smaller datasets.

In January 2014, a multi-institutional collaborative project was initiated in Italy between accredited thoracic surgery programs, with the aim of prospectively collecting data from VATS lobectomy operations. Specific data on the management of LN during surgery was included in the database and has now become available for analysis.

The availability of a large bulk of detailed information on LN management during VATS lobectomy has prompted us to analyze the impact of different nodal resection strategies and to assess if one or more predictors of an adequate $\mathrm{LN}$ management could be identified in our population.

\section{Methods}

The Italian VATS Group registry database contains prospectively collected data on VATS lobectomies performed since January $1^{\text {st }}, 2014$ across 56 Italian certified thoracic surgery centers. All VATS lobectomies included in the database between $1 / 1 / 2014$ and 10/21/2016 were considered for the analysis. Patients' records included age, sex, Charlson and Eastern Cooperative Oncology Group (ECOG) comorbidity score $(16,17)$, and the technique of VATS lobectomy. The pre-operative clinical stage was determined based on the results of pre-operative CT scan, PET scan, mediastinoscopy, and endoscopic bronchial or esophageal ultrasound (EBUS, EUS). The final postoperative pathology report was available for all patients. The type of surgical technique used for the intra-operative management of hilar and mediastinal nodes was recorded (complete dissection or SS). Specific data on intra-operative $\mathrm{LN}$ management was also recorded, including the number of $\mathrm{LN}$ resected (RN), the number of positive $\mathrm{LN}$, and the number and type of positive LN stations (LNS). A LNR (number of positive LN/number of LN resected) (LNR) was also calculated from the dataset.

The complication rates and early (30- and 90-day) survival were also analyzed. Complications were recorded according to the Dindo (18) classification and analyzed using a multivariate model to assess the impact of LNrelated variables.

Given the relatively short follow/up time, long-term overall and disease-free survival figures were not available yet for this population. Nodal upstaging was used as a predictor of the efficacy of intra-operative LN management and was the main outcome variable of the study. N0-to-N1, $\mathrm{N} 0$-to-N2, and N1-to-N2 upstaging was recorded and analyzed.

The aim of the study was to identify the intra-operative LN management strategy showing the best correlation with nodal upstaging. CD was compared with SS and with a 
quantitative assessment of the number of LNR, LNS, type of positive stations and LNR.

In order to summarize the most relevant features of the clinical variables, descriptive statistics were performed. Categorical variables are presented using the distribution of absolute frequencies (percentages and cumulative); continuous variables are presented as mean, standard deviation, median, max, min, and range. Data from the Italian VATS Group registry was retrieved using a specific query tool on 10/21/2016. The study was peer-reviewed and approved by the Italian VATS Group scientific advisory board. All the patients included in the prospective collection of data within the Italian VATS group study had signed an informed consent and the VATS group registry database project underwent approval by the IRRB of each participating center.

Continuous variables were reported as the mean \pm standard deviation, whereas categorical variables were given as percentages. Fisher's exact tests were used to compare dichotomous data, and Pearson's $\chi^{2}$ for categorical variables. Proportional hazards assumptions were analytically assessed using Schoenfeld residuals. Violations were addressed by the inclusion of time-varying co-variable. Univariate Logistic Regression was performed to evaluate any association between dependent and independent variables. ROC analysis was performed to assess cut-off points that provided the best discriminating capacity for quantitative parameters. To overcome the over-dispersion caused by a Generalized Linear Model (GLM) with Poisson distribution we used a GLM with negative binomial distribution for LN count and for positive stations count. A fractional model was used for the $\mathrm{N} 1$ and $\mathrm{N} 2$ ratio variables. A two tailed $\mathrm{P}$ value below 0.05 was considered significant. Statistical analysis was performed using SAS 9.4 (SAS Institute Inc., Cary, NC, USA) and STATA package version 14 (STATA Corp LP, College, TX).

All the patients gave informed consent for participation to the study. The study was approved by the Ismett Institutional Research Review Board (IRRB) with the ID number IRRB/39/14.

\section{Results}

Within the above-mentioned time frame, 3,219 cases of VATS lobectomy were enrolled in the database. $58 \%$ of the patients were males and $42 \%$ were females. The mean age at the time of the operation was $67.6 \pm 9.02$ years (range: 18-89 years). The average patient Charlson and
ECOG comorbidity scores were $4.40 \pm 1.82$ (range: $0-15$ ) and $0.27 \pm 0.52$ (range: $0-4$ ), respectively. An "anterior" (biportal or triportal) surgical approach was used for VATS lobectomy in $85.38 \%$ of the cases. Other approaches (posterior, lateral, totally endoscopic, uniportal) were used in the remaining $14.62 \%$ of cases. An upper lobectomy was the most frequent operation performed (56.2\% of all cases) and the right side was more frequently operated than the left $(61.4 \%$ vs. $38.6 \%$ of the cases). Most of the cases had a diagnosis of adenocarcinoma $(73.5 \%)$ or squamous cell carcinoma $(15.3 \%)$ of the lung.

Ninety eight VATS lobectomies performed for benign disease and 233 vats lobectomies performed for malignant disease other than "non-small cell lung cancer" (NSCLC) were excluded from the analysis. Also, patients who underwent neo-adjuvant treatment $(\mathrm{N}=94)$ and patients who required conversion from VATS to thoracotomy $(\mathrm{N}=298)$ were excluded from the analysis, in order to avoid possible confounders.

Based on the pre-operative CT scan assessment, 2,423 patients had early stage, clinical stage I or stage II NSCLC. 2,360 patients had a clinical evidence of N0 disease $(97.4 \%)$ while 63 had N1 disease (2.6\%). A preoperative PET scan was available in 2,216 NSCLC patients (91.4\%), while pre-operative invasive staging of the LN (mediastinoscopy, EBUS or EUS) was performed in 86 patients $(3.5 \%)$.

668 patients underwent intra-operative SS $(27.7 \%)$ and 1743 complete dissection $(72.3 \%)$ of all hilar and mediastinal LN. An average of $6.23 \pm 4.15 \mathrm{~N} 1 \mathrm{LN}$ (range: $0-35$ ) and 7.18 $\pm 5.55 \mathrm{~N} 2 \mathrm{LN}$ (range: 0-49) were resected during surgery (Table 1). The average ratio of positive/ resected $\mathrm{LN}$ was 0.04 for $\mathrm{N} 1 \mathrm{LN}$ and 0.02 for N2 LN. The average number of positive $\mathrm{LN}$ stations was $1.63 \pm 0.98$ (range: $1-7$ ): $1.22 \pm 0.45 \mathrm{~N} 1$ (range: $1-3$ ) and $1.34 \pm 0.64 \mathrm{~N} 2$ (range: 1-5).

Overall, nodal upstaging occurred in 292 (12.05\%) patients. N0-to-N1 nodal upstaging occurred in 146 cases (6.03\%), N0-to-N2 in 132 cases (5.45\%). N1-to-N2 upstaging was observed in 14 cases $(0.58 \%)$ (Table 2).

Patients undergoing complete dissection had, in average, more $\mathrm{N} 1 \mathrm{LN}$ resected $(6.56$ vs. $5.38, \mathrm{P}<0.001)$, but a similar N1 LNR (0.04 vs. 0.03; $\mathrm{P}=0.151)$ compared to patients undergoing SS.

Patients undergoing $\mathrm{CD}$ had also more $\mathrm{N} 2 \mathrm{LN}$ resected (7.93 vs. 5.24, $\mathrm{P}<0.001)$ and a slightly higher $\mathrm{N} 2 \mathrm{LNR}(0.03$ vs. $0.02 ; \mathrm{P}=0.024)$ compared to patients undergoing SS.

The average number of positive $\mathrm{LN}$ stations was 1.68 in 


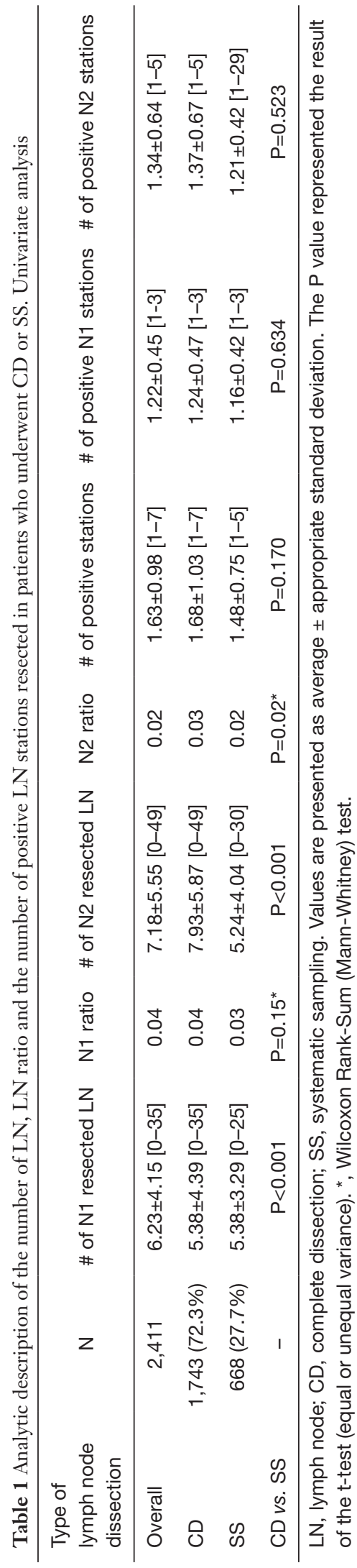

patients undergoing $\mathrm{CD} v s .1 .48$ in patients undergoing SS $(\mathrm{P}=0.170)$. There was no statistically significant difference in the number of positive $\mathrm{N} 1$ and $\mathrm{N} 2$ retrieved $\mathrm{LN}$ stations between patients undergoing CD or SS (1.24 vs. 1.16 and 1.37 vs. 1.21 , respectively; $\mathrm{P}=0.634$ and 0.523$)$.

After univariate analysis, no difference could be appreciated in the rate of $\mathrm{N} 0$-to- $\mathrm{N} 1$ and $\mathrm{N} 0-$ to- 2 upstaging based solely on the type of surgical technique used for the intraoperative management of $\mathrm{LN}$ (CD or $\mathrm{SS})$. The $\mathrm{N} 0$-to-N1 upstaging rate was $5.9 \%$ in patients undergoing $\mathrm{CD}$ vs. $6.4 \%$ in patients undergoing SS $(\mathrm{P}=0.627)$. The $\mathrm{N} 0$-to-N2 upstaging rate was $5.9 \%$ in patients undergoing $\mathrm{CD}$ vs. $4.2 \%$ in patients undergoing $\mathrm{SS}$ $(\mathrm{P}=0.086)$. N1-to-N2 upstaging was observed exclusively in patients undergoing CD (19 patients) (Table 2).

On logistic regression, the overall number of resected nodes was related with both $\mathrm{N} 0$-to- $\mathrm{N} 1(\mathrm{OR}=1.02$, CI, 1.01-1.04; $\mathrm{P}=0.03$ ) and $\mathrm{N} 0$-to-N2 upstaging (OR $=1.03 ; \mathrm{CI}, 1.01-1.05 ; \mathrm{P}=0.001$ ) (Table 3). The ROC analysis showed that resection of 12 nodes was the best predictor of $\mathrm{N} 1$ upstaging with an Area under the curve (AUC) of ROC of 0.56 (N0-to-N2), while 11 nodes was the best predictor of N0-to-N2 upstaging with an AUC-ROC of 0.59.

The number of resected $\mathrm{N} 1$ nodes correlated with $\mathrm{N} 0$ to-N1 upstaging (OR =1.07; CI, 1.04-1.11, $\mathrm{P}<0.001)$ but not with $\mathrm{N} 0$-to-N2 (OR $=1.02$; $\mathrm{CI}, 0.98-1.06 ; \mathrm{P}=0.282)$ nor N1-to-N2 (OR =0.99; CI, 0.87-1.13; $\mathrm{P}=0.944)$ upstaging. Resection of $6 \mathrm{~N} 1$ nodes had the best discriminatory performance to predict N0-to-N1 upstaging, with an AUCROC of 0.63 .

The number of resected $\mathrm{N} 2$ nodes correlated with $\mathrm{N} 0$ to-N2 (OR =1.05; CI, 1.02-1.07, $\mathrm{p}<0.001)$ and N1-to-N2 upstaging ( $\mathrm{OR}=1.07$; $\mathrm{CI}, 1.02-1.14 ; \mathrm{P}=0.008)$ but not with $\mathrm{N} 0$-to-N1 upstaging ( $\mathrm{OR}=0.99 ; \mathrm{CI}, 0.96-1.02 ; \mathrm{P}=0.665)$. Resection of $7 \mathrm{~N} 2$ nodes had the best discriminatory performance to predict N0-to-N2 upstaging, with an AUCROC of 0.60 .

The number of positive nodal stations was correlated with $\mathrm{N} 0$-to-N1, N0-to-N2 and N1-to $\mathrm{N} 2$ upstaging, with an $\mathrm{OR}$ of 3.01, 13.81, and 3.57, respectively. The best discriminatory performance to predict N0-to-N1 upstaging was the finding of 1 positive LN node station (AUC-ROC $=0.94$ ). The best discriminatory performance to predict $\mathrm{N} 0$-to-N2 and N1-to-N2 upstaging was the finding of two positive LN stations (AUC-ROC $=0.98$ and 0.97 , respectively, Figure 1).

The number of positive N1 LNS had also significant correlation with $\mathrm{N} 0$-to- $\mathrm{N} 2(\mathrm{OR}=6.83, \mathrm{AUC}=0.75$, 
Table 2 Rates of upstaging in patients undergoing complete dissection (CD) or systematic sampling (SS)

\begin{tabular}{lcccc}
\hline Type of lymph node dissection & $\mathrm{N}$ & Upstage (N0-N1) & Upstage (N0-N2) & Upstage (N1-N2) \\
\hline Overall & 2,411 & $146(6.03 \%)$ & $132(5.45 \%)$ & $14(0.58 \%)$ \\
CD & $1,743(72.3 \%)$ & $103(5.9 \%)$ & $104(5.9 \%)$ & $14(0.80 \%)$ \\
SS & $668(27.7 \%)$ & $43(6.4 \%)$ & $28(4.2 \%)$ & 0 \\
CD vs. SS & - & $P=0.627$ & $P=0.086$ & $P=0.020$ \\
\hline
\end{tabular}

Table 3 Impact on nodal upstaging of the number of lymph nodes, lymph node ratio and the number of positive nodal stations resected. Multivariate analysis

\begin{tabular}{|c|c|c|c|c|c|c|}
\hline Upstage & Overall \# of resected LN & \# N1 resected LN & \# N2 resected LN & N1 LN ratio & N2 LN ratio & Overall \# of positive stations \\
\hline OR & 1.02 & 1.07 & 0.99 & & 0.26 & 3.01 \\
\hline $95 \% \mathrm{Cl}$ & $1.01-1.04$ & $1.04-1.11$ & $0.96-1.02$ & & $0.03-2.44$ & $2.53-3.58$ \\
\hline $\mathrm{AUC}[\mathrm{N}]$ & $0.56[12]$ & $0.63[6]$ & & $0.95[0.07]$ & & $0.94[1]$ \\
\hline \multicolumn{7}{|l|}{ N0-N2 } \\
\hline OR & 1.03 & 1.02 & 1.05 & & & 13.81 \\
\hline $95 \% \mathrm{Cl}$ & $1.01-1.05$ & $0.98-1.06$ & $1.02-1.07$ & & & $10.2-18.7$ \\
\hline $\mathrm{AUC}[\mathrm{N}]$ & $0.59[11]$ & & $0.60[7]$ & $0.74[0.06]$ & $0.97(0.07)$ & $0.98[2]$ \\
\hline OR & 1.04 & 0.99 & 1.08 & & & 3.57 \\
\hline $95 \% \mathrm{Cl}$ & $0.99-1.09$ & $0.87-1.13$ & $1.02-1.14$ & & & $2.58-4.93$ \\
\hline $\mathrm{AUC}[\mathrm{N}]$ & 0.67 [13] & & $0.73[7]$ & $0.86[0.08]$ & $0.96[0.08]$ & $0.97[2]$ \\
\hline$P$ value & 0.064 & 0.944 & 0.008 & & & $<0.001$ \\
\hline
\end{tabular}

LN, lymph node; CD, complete dissection; SS, systematic sampling; OR, odds ratio; Cl, confidence interval; AUC, area under the curve.

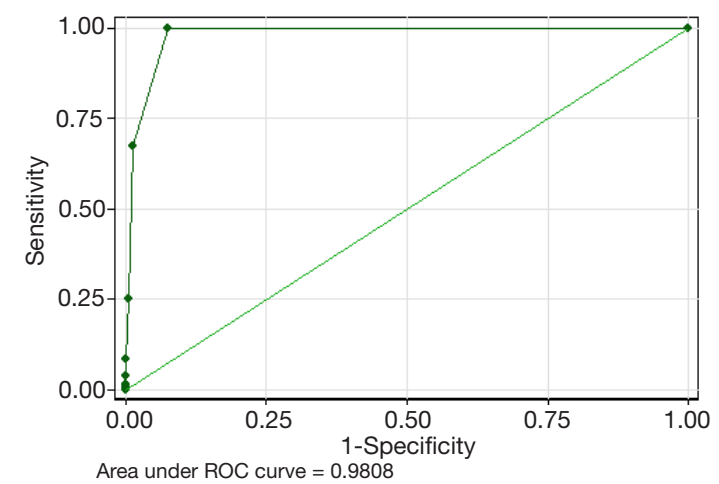

Figure 1 ROC analysis of the impact of the number of positive LN stations resected on N0-to-N2 upstaging. ROC, receiver operating characteristic; LN, lymph node.
$\mathrm{P}<0.001)$ and $\mathrm{N} 1$-to-N2 $(\mathrm{OR}=7.49, \mathrm{AUC}=0.83, \mathrm{P}<0.001)$.

An analysis of each single positive $\mathrm{LN}$ station resected was also performed to assess any potential relationship with nodal upstaging. Each single positive N1 station correlated well with N1 upstaging and each single positive N1 and N2 stations correlated well with $\mathrm{N} 2$ upstaging. We were not able to find any role for one single $\mathrm{LN}$ station in predicting the rates of upstaging.

Interestingly, the preoperative $\mathrm{T}$ staging did not correlate with any upstaging. The final, pathologic $\mathrm{T}$ stage instead was a significant predictor of both N0-to-N1 (T2) and N0-to-N2 (T2-T3-T4) upstaging (Table 4).

To assess the influence of different pre-operative staging methods on the results of intraoperative LN management, 
Table 4 Correlation between $\mathrm{T}$ staging and upstaging rates

\begin{tabular}{|c|c|c|c|c|}
\hline T Stage & N (\%) & N0 to N1 (\%) & N0 to N2 (\%) & N1 to N2 (\%) \\
\hline $\mathrm{T} 1$ & $1,843(76.1)$ & $101(5.5)$ & $94(5.1)$ & $11(0.6)$ \\
\hline $\mathrm{T} 2$ & $536(22.1)$ & 41 (7.6); $P=0.065$ & 34 (6.3); $P=0.263$ & $3(0.5) ; P=0.921$ \\
\hline T3 & $43(1.8)$ & 3 (6.9); $P=0.672$ & 4 (9.3); $P=0.228$ & 0 \\
\hline $\mathrm{T} 1$ & $1,514(62.5)$ & $77(5.1)$ & $53(40.1)$ & $6(0.4)$ \\
\hline $\mathrm{T} 2$ & 790 (32.6) & 55 (6.9); $P=0.07$ & 64 (48.5); $P<0.0001$ & $6(0.7) ; P=0.259$ \\
\hline T3 & $105(4.3)$ & 13 (12.4); $P=0.002$ & 13 (9.8); $P<0.0001$ & 1 (0.9); $P=0.416$ \\
\hline T4 & $14(0.6)$ & 1 (7.1); $P=0.729$ & 2 (14.3); $P=0.05$ & 1 (7.1); $P=0.08$ \\
\hline
\end{tabular}

Table 5 Type and occurrence of complications related to different LN management and lymph node-related variables during VATS lobectomy

\begin{tabular}{|c|c|c|c|c|c|c|c|c|c|c|c|c|}
\hline \multirow[t]{2}{*}{ Observed variable } & \multicolumn{2}{|c|}{ Atrial fibrillation } & \multicolumn{2}{|c|}{ Pneumonia } & \multicolumn{2}{|c|}{ Hemothorax } & \multicolumn{2}{|c|}{ Chylothorax } & \multicolumn{2}{|c|}{$\begin{array}{l}\text { Phrenic nerve } \\
\text { injury }\end{array}$} & \multicolumn{2}{|c|}{$\begin{array}{c}\text { Laryngeal nerve } \\
\text { palsy }\end{array}$} \\
\hline & I-II & $>11$ & I-II & $>11$ & I-II & $>$ II & I-II & $>11$ & I-II & $>$ II & $I-I I$ & $>11$ \\
\hline Upstage N0-N1 & \multicolumn{2}{|c|}{0.06} & \multicolumn{2}{|c|}{0.595} & \multicolumn{2}{|c|}{$<0.001$} & \multicolumn{2}{|c|}{0.859} & \multicolumn{2}{|c|}{0.670} & \multicolumn{2}{|c|}{0.757} \\
\hline \# LNR & 0.035 & 0.470 & 0.037 & 0.466 & 0.963 & 0.453 & 0.095 & 0.036 & 0.028 & $\mathrm{~N} / \mathrm{A}$ & 0.992 & $\mathrm{~N} / \mathrm{A}$ \\
\hline \# N1 LNR & 0.080 & 0.413 & 0.834 & 0.656 & 0.864 & 0.785 & 0.621 & 0.033 & 0.080 & N/A & 0.318 & $\mathrm{~N} / \mathrm{A}$ \\
\hline N1 LNR & 0.513 & 0.991 & 0.791 & 0.632 & 0.561 & 0.027 & \multicolumn{2}{|c|}{ N/A } & \multicolumn{2}{|c|}{ N/A } & 0.579 & N/A \\
\hline \# Pos. N1 LN station & 0.367 & 0.981 & 0.364 & 0.749 & 0.988 & 0.005 & & & $\mathrm{~N}$ & & 0.365 & N/A \\
\hline \# Pos. N2 LN station & 0.901 & 0.988 & 0.861 & 0.046 & & & 0.433 & 0.997 & $\mathrm{~N}$ & & 0.014 & N/A \\
\hline
\end{tabular}

All values are presented as "P" and are significant for $P$ values $<0.05$. LN, lymph node; LNR, lymph node ratio; N/A, not available.

we applied the model of the study to the sub-population of patients who received both a pre-operative CT scan and PET scan $(\mathrm{N}=2,216)$. Both the overall and specific ( $\mathrm{N} 1$ or N2) upstaging rates were similar to the figures of the population who received staging with a CT scan only.

The occurrence of some complications after VATS lobectomy in some cases could be related to the modality of lymph-node management (Table 5). The number of resected LN correlated significantly with the occurrence of atrial fibrillation, pneumonia, chylothorax and phrenic nerve injury. Postoperative hemothorax was more frequent in patients with N0-to-N1 upstage, higher N1 LN rate, higher number of $\mathrm{LN}$ stations.

\section{Discussion}

The optimal intra-operative nodal management during lobectomy for early stage NSCLC has been debated for 
decades. The importance of obtaining nodes for pathologic examination relies on the fact that the final pathologic $\mathrm{N}$ stage is strictly related to overall and disease-free survival.

Darling et al. explored the differences between CD and SS during lobectomy (6). The study suggested similar outcomes between the two approaches, and justified the use of SS as a less invasive but oncologically equivalent strategy for the intra-operative management of LN. In this study, including an intraoperative $\mathrm{LN}$ frozen section before randomization may have not reflected the standard clinical practice, as noted by Cerfolio (19).

Criticism to this type of analysis led to introducing more quantitatively reliable parameters for the assessment of $\mathrm{LN}$ resection during lobectomy.

Zhou and colleagues retrospectively reviewed the results of a single-center experience on 493 patients with early stage lung cancer undergoing VATS lobectomy or segmentectomy (20). They were able to show that sampling greater than $3 \mathrm{LN}$ stations and greater than $10 \mathrm{LN}$ was associated with an increase in nodal upstaging, and that sampling 3 or more $\mathrm{LN}$ stations was an independent predictor of mortality. These results suggest the feasibility of using a measurable intra-operative $\mathrm{LN}$ resection strategy. The study had the limitation of including both lobectomies and segmentectomies, of being a single-center series and of limiting the analysis to less than 500 patients.

Several authors have criticized a "quantitative" approach to the assessment of intraoperative LN management, mostly because of the inter-personal variability of the number of nodes. Riquet and colleagues (21) reviewed data from 1,095 patients across two French centers and recognized a great variability of the number of nodes retrieved during lung resections, following a "Gaussian" distribution. They did not find any correlation between the number of resected nodes and patients' outcome, and therefore strived the importance of resecting all the hilar and mediastinal nodal stations. The study included patients undergoing different types of lung resections, at all stages of disease.

Another pitfall of a quantitative assessment of LN resection is the variability of the pathologic evaluation of the number of nodes resected. Fragments of LN may be recorded as whole $\mathrm{LN}$ and the pathologist's assessment may not be uniform across different institutions.

VATS has been recognized as an established surgical technique for the treatment of early stage lung cancer. The oncological outcomes of VATS lobectomy have been shown to be equal or superior to those of open lobectomy. The VATS approach has the advantage of less postoperative pain, faster post-operative patient recovery and discharge home, and a faster access to post-operative adjuvant treatments in case these are needed $(2,3)$. Several studies have shown the feasibility and safety of an adequate $\mathrm{LN}$ resection using all VATS lobectomy techniques (13-15).

In Italy, in 2014, a large collaborative study on VATS lobectomy ("VATS Group") was established and a prospective, multi-centric database was started. In our study, we identified a large population of patients with clinical early-stage NSCLC with a considerable amount of information on the management of $\mathrm{LN}$, although lacking survival rates.

Most of the patients had a pre-operative CT scan and PET scan $(90.7 \%)$. Only a minority of the patients had an invasive LN staging, either mediastinoscopy, EBUS or EUS. The availability of the PET scan for most patients with early-stage disease justify these findings. Also, the VATS Group recommends following the ESTS guidelines (22), suggesting invasive LN staging in tumors larger than $3 \mathrm{~cm}$, centrally located or with positive PET findings.

For most of the cases, the surgeon reported performing a complete nodal dissection $(72.1 \%)$. This finding is also consistent with a broad acceptance and application of the European guidelines.

The average number of nodes retrieved during surgery matched those of other similar series in the literature: in average, $6.23 \mathrm{~N} 1$ and $7.18 \mathrm{~N} 2$ nodes were retrieved.

The nodal upstaging rate of $12.06 \%$ was slightly similar to the results of other studies. Licht et al. reported nodal upstaging in $11.9 \%$ of patients in their report from the Danish VATS lobectomy registry including 717 patients with stage I NSCLC (12). Boffa and colleagues reported an upstaging rate of $11.6 \%$ in a retrospective analysis of the STS database on 4,394 patients (14).

Our rates of nodal upstaging compare well with the rates reported from other large multicenter databases such as the National Cancer Database (12.8\% for open lobectomies vs. 10.3 for VATS). Medbery and colleagues suggested that the lower upstaging rates observed for VATS cases might result from non-academic or non-research thoracic surgery divisions (23). Our database might be a source for further assessment of the relationship between surgeon's experience and accuracy of lymphadenectomy, by using specific and dedicated tools.

The report of very low upstaging rates for VATS lobectomy (down to $4.8 \%$ ), such as in the study by Martin et al. (24), who reviewed a state cancer database from Kentucky, might hold a significant selection bias and give an 
altered perception of the role of VATS and on the adequacy of VATS lobectomy as a reliable oncologic procedure. Given the difficulty to collect adequate data on this topic, the relevance of the results obtained from a dedicated, multi-centric database such as the VATS Group registry should be further emphasized.

As expected, patients undergoing CD had a statistically significant higher number of $\mathrm{LN}$ resected compared to those undergoing SS, both $\mathrm{N} 1$ (6.61 vs. 5.38,) and N2 (7.93 vs. 5.24). Nevertheless, this finding translated into an equal LNR for N1 nodes and only in a minimally higher LNR for N2 nodes. Similarly, the number of positive LNS was not statistically different between the CD and SS group.

The above findings are further supported by the fact that, in our series, the nodal upstaging rates were not significantly different between patients undergoing CD or SS, suggesting no evident benefit for one or the other approach.

On the other side, we were able to correlate the rates of nodal upstaging to the number of $\mathrm{LN}$ resected (both $\mathrm{N} 1$ and N2). N1 and N2 upstaging were more frequent in patients who had 12 or more LN resected, as indicated by the ROC-AUC analysis. Resection of $\geq 6 \mathrm{~N} 1$ or $\geq 7 \mathrm{~N} 2$ nodes was the threshold to obtain significant correlation with N0-to-N1 or N0-to-N2 upstaging, respectively. These figures strongly suggest that it may possible to discriminate between patients who will have nodal upstaging based on the number of resected $\mathrm{LN}$, independently from the surgical technique. It is our expectation that, with the availability of more data, despite interpersonal variability, it may be eventually possible to set a minimum number of nodes to be resected to prevent inadequate staging.

Although our database lacked information about the number of LN stations resected, we had the opportunity to examine the number of positive $\mathrm{LN}$ stations resected. In general, the finding of at least one positive nodal station could predict N0-to-N1 upstaging, while the finding of at least two positive nodal stations was correlated to N0to-N2 upstaging $(\mathrm{OR}=3.01$ and $13.8, \mathrm{P}<0.0001)$. More interestingly, positive $\mathrm{N} 1$ nodal stations correlated with N0-to-N2 and N1-to-N2 upstaging. This may suggest that, during surgery, the presence of suspicious N1 nodes should mandate an aggressive $\mathrm{N} 2$ dissection in order to obtain an adequate nodal resection and staging.

We were interested in assessing if one specific positive LN station correlated with nodal upstaging, but this phenomenon was not observed in our series. Resecting one specific $\mathrm{LN}$ station cannot therefore be used as a viable tool to guide the intraoperative resection of $\mathrm{LN}$.

Restricting the analysis to patients undergoing a more aggressive pre-operative staging didn't change the figures of nodal upstaging. These findings suggest that CT/PET allows in most cases a reasonable pre-operative staging in a selected population of patients with stage I-II NSCLC.

The role of tumor size and location is also reported to play a significant role in the nodal upstaging rates in many papers comparing open and VATS lobectomy, as pointed out by Kocher et al. (25). In our VATS series, the proportion of T2 and T3 lesions was very small, as expected. Nevertheless, on logistic regression we could find a significant correlation between larger lesions and the N0-to-N1 (T3) and N0-to-N2 (T1, 2, 3) upstaging rates. This observation seems to justify the role of VATS lymph-node resections even for larger and centrally located lesions.

Some complications of VATS lobectomy could be related to specific approaches to lymph-node resection. While performing lymph-node dissection during lobectomy, the surgeon should be aware about the risk of complications such as atrial fibrillation, pneumonia, chylothorax, hemothorax and nerve injury. This study was not focused on the analysis of complications and further work is desirable on this topic.

As already mentioned, the study has some limitations. Due to the short follow-up period, survival rates were not yet available for this population. Nodal upstaging was used as a surrogate marker of the adequacy of intraoperative $\mathrm{LN}$ treatment and, indirectly, of the outcome of patients.

The possibility of sampling errors during the pathologic examination of the specimens may account for possible bias in assessing the number of nodes resected. Standardization or centralization of the pathology examination would improve the accuracy of the analysis.

In conclusion, we examined a large dataset of VATS lobectomies with specific data on intra-operative LN management. Our study compared the impact of different intraoperative approaches (CD/SS) to a more quantitative assessment of $\mathrm{LN}$ resected.

Performing CD or SS did not impact "per se" on the rates of nodal upstaging. Resecting a minimal number of nodes (12 overall or 6/7 for individual N1 or N2 levels) seems a promising approach to optimize the chances that nodal upstaging is not missed. Finding obviously pathologic LN stations during dissection should prompt at a more aggressive dissection due to the increased probability of upstaging. The availability of survival figures is needed to confirm the suggested findings. 


\section{Acknowledgements}

None.

\section{Footnote}

Conflicts of Interest: The authors have no conflicts of interest to declare.

Ethical Statement: All the patients gave informed consent for participation to the study. The study was approved by the Ismett Institutional Research Review Board (IRRB) with the ID number IRRB/39/14.

*, List of collaborators of the Italian VATS Group: Carlo Curcio, MD (Monaldi Hospital, Napoli); Dario Amore, MD (Monaldi Hospital, Napoli); Giuseppe Marulli, MD (University of Padova); Samuele Nicotra, MD (University of Padova); Andrea De Negri, MD (San Martino Hospital, Genova); Paola Maineri, MD (San Martino Hospital, Genova); Gaetano di Rienzo (Vito Fazzi Hospital, Lecce); Camillo Lopez, MD (Vito Fazzi Hospital, Lecce); Angelo Morelli, MD (S. Maria delle Misericordia Hospital, Udine); Francesco Londero, MD (S. Maria delle Misericordia Hospital, Udine); Lorenzo Spaggiari, MD (IEO Hospital, Milano); Roberto Gasparri, MD (IEO Hospital, Milano); Guido Baietto, MD (Maggiore della Carità Hospital, Novara); Caterina Casadio, MD (Maggiore della Carità Hospital, Novara); Maurizio Infante, MD (Borgo Trento Hospital, Verona); Cristiano Benato, MD (Borgo Trento Hospital, Verona); Marco Alloisio, MD (IRCCS Humanitas, Milano); Edoardo Bottoni, MD (IRCCS Humanitas, Milano); Giuseppe Cardillo, MD (Forlanini Hospital, Roma); Francesco Carleo, MD (Forlanini Hospital, Roma); Franco Stella, MD (S. Orsola Hospital, Bologna); Giampiero Dolci, MD (S. Orsola Hospital, Bologna); Francesco Puma, MD (University of Perugia); Damiano Vinci, MD (University of Perugia); Giorgio Cavallesco, MD (University of Ferrara); Pio Maniscalco, MD (University of Ferrara); Luca Ampollini, MD (University of Parma); Paolo Carbognani, MD (University of Parma); Alberto Terzi, MD (Negrar Hospital, Verona); Andrea Viti, MD (Negrar Hospital, Verona); Giampiero Negri, MD (S. Raffaele Hospital, Milano); Alessandro Bandiera, MD (S. Raffaele Hospital, Milano); Reinhold Perkmann, MD (Bolzano Hospital, Bolzano); Francesco Zaraca, MD (Bolzano Hospital, Bolzano); Claudio Andretti, MD (S. Andrea Hospital, Roma); Camilla Poggi, MD (S. Andrea Hospital,
Roma); Felice Mucilli, MD (S. Maria Annunziata Hospital, Chieti); Pierpaolo Camplese, MD (S. Maria Annunziata Hospital, Chieti); Luca Luzzi, MD (University of Siena); Marco Ghisalberti, MD (University of Siena); Andrea Imperatori, MD (University of Varese); Nicola Rotolo, MD (University of Varese); Luigi Bortolotti, MD (Humanitas Gavazzeni Hospital, Bergamo); Giovanna Rizzardi, MD (Humanitas Gavazzeni Hospital, Bergamo); Massimo Torre, MD (Niguarda Hospital, Milano); Alessandro Rinaldo, MD (Niguarda Hospital, Milano); Armando Sabbatini , MD (Ospedali Riuniti, Ancona); Majed Refai, MD (Ospedali Riuniti, Ancona); Mauro Roberto Benvenuti, MD (Spedali Civili, Brescia); Diego Benetti, MD (Spedali Civili, Brescia); Alessandro Stefani, MD (Ospedale Policlinico, Modena); Pamela Natali, MD (Ospedale Policlinico, Modena); Paolo Lausi, MD (Ospedale Molinette, Torino); Francesco Guerrera, MD (Ospedale Molinette, Torino).

\section{References}

1. Detterbeck FC, Postmus PE, Tanoue LT. The stage classification of lung cancer: Diagnosis and management of lung cancer, 3rd ed: American College of Chest Physicians evidence-based clinical practice guidelines. Chest 2013;143:e191S-210S.

2. McKenna RJ Jr, Houck W, Fuller CB. Video-assisted thoracic surgery lobectomy: experience with 1,100 cases. Ann Thorac Surg 2006;81:421-5; discussion 425-6.

3. Takagi H, Matsui M, Umemoto T. Long-term survival of VATS versus open lobectomy. Ann Thorac Surg 2011;92:408-9; author reply 409-10.

4. Rusch VW, Giroux DJ. Nodal staging in lung cancer: lymph node location or number? J Thorac Oncol 2011;6:237-8.

5. van Velzen E, Snijder RJ, Brutel de la Rivière A, et al. Type of lymph node involvement influences survival rates in T1N1M0 non-small cell lung carcinoma. Lymph node involvement by direct extension compared with lobar and hilar node metastases. Chest 1996;110:1469-73.

6. Darling GE, Allen MS, Decker PA, et al. Randomized trial of mediastinal lymph node sampling versus complete lymphadenectomy during pulmonary resection in the patient with N0 or N1 (less than hilar) non-small cell carcinoma: results of the American College of Surgery Oncology Group Z0030 Trial 2011;141:662-70.

7. Ludwig MS, Goodman M, Miller DL, et al. Post-operative survival and the number of lymph nodes sampled during resection of node-negative non-small cell lung cancer. 
Chest 2005;128:1545-50.

8. Nwogu CE, Groman A, Fahey D, et al. Number of lymph nodes and metastatic lymph node ratio are associated with survival in lung cancer. Ann Thorac Surg 2012;93:1614-9; discussion 1619-20.

9. Jonnalagadda S, Smith C, Mhango G, et al. The number of lymph node metastases as a prognostic factor in patients with $n 1$ non-small cell lung cancer. Chest 2011;140:433-40.

10. Jonnalagadda S, Arcinega J, Smith C, et al. Validation of the lymph node ratio as a prognostic factor in patients with N1 non small cell lung cancer. Cancer 2011;117:4724-31.

11. Wisnivesky JP, Arciniega J, Mhango G, et al. Lymph node ratio as a prognostic factor in elderly patients with pathological $\mathrm{n} 1$ non-small cell lung cancer. Thorax 2011;66:287-93.

12. Licht PB, Jørgensen OD, Ladegaard L, et al. A national study of nodal upstaging after thoracoscopic versus open lobectomy for clinical stage I lung cancer. Ann Thorac Surg 2013;96:943-9; discussion 949-50.

13. D'Amico TA, Niland J, Mamet R, et al. Efficacy of mediastinal lymph node dissection during lobectomy for lung cancer by thoracoscopy and thoracotomy. Ann Thorac Surg 2011;92:226-31; discussion 231-2.

14. Boffa DJ, Kosinski AS, Paul S, et al. Lymph node evaluation by open or video-assisted approaches in 11,500 anatomic lung cancer resections. Ann Thorac Surg 2012;94:347-53

15. Ramos R, Girard P, Masuet C, et al. Mediastinal lymph node dissection in early-stage non-small cell lung cancer: totally thoracoscopic vs thoracotomy. Eur J Cardiothorac Surg 2012;41:1342-8.

16. Charlson ME, Pompei P, Ales KL, et al. A new method of classifying prognostic comorbidity in longitudinal studies: development and validation. J Chronic Dis 1987;40:373-83.

17. Oken MM, Creech RH, Tormey DC, et al. Toxicity and response criteria of the Eastern Cooperative Oncology

Cite this article as: Bertani A, Gonfiotti A, Nosotti $M$, Ferrari PA, De Monte L, Russo E, Di Paola G, Solli P, Droghetti A, Bertolaccini L, Crisci R; Italian VATS Group. Nodal management and upstaging of disease: initial results from the Italian VATS Lobectomy Registry. J Thorac Dis 2017;9(7):2061-2070. doi: 10.21037/jtd.2017.06.12
Group. Am J Clin Oncol 1982;5:649-55.

18. Dindo D, Demartines N, Clavien PA. Classification of surgical complications: a new proposal with evaluation in a cohort of 6336 patients and results of a survey. Ann Surg 2004;240:205-13.

19. Cerfolio RJ, Bryant AS, Minnich DJ. Complete thoracic mediastinal lymphadenectomy leads to a higher rate of pathologically proven N2 disease in patients with nonsmall cell lung cancer. Ann Thorac Surg 2012;94:902-6.

20. Zhou H, Tapias LF, Gaissert HA, et al. Lymph Node Assessment and Impact on Survival in Video-Assisted Thoracoscopic Lobectomy or Segmentectomy. Ann Thorac Surg 2015;100:910-6.

21. Riquet $M$, Legras A, Mordant $P$, et al. Number of mediastinal lymph nodes in non-small cell lung cancer: a Gaussian curve, not a prognostic factor. Ann Thorac Surg 2014;98:224-31.

22. Lardinois D, De Leyn P, Van Schil P, et al. ESTS guidelines for intraoperative lymph node staging in nonsmall cell lung cancer. European Journal of Cardiothoracic Surgery 2006;30:787-92.

23. Medbery RL, Gillespie TW, Liu Y, et al. Nodal Upstaging Is More Common with Thoracotomy than with VATS During Lobectomy for Early-Stage Lung Cancer: An Analysis from the National Cancer Data Base. J Thorac Oncol 2016;11:222-33.

24. Martin JT, Durbin EB, Chen L, et al. Nodal Upstaging During Lung Cancer Resection Is Associated With Surgical Approach. Ann Thorac Surg 2016;101:238-44; discussion 44-5.

25. Kocher F, Ng C, Augustin F. On the Article "Nodal Upstaging Is More Common with Thoracotomy Than with VATS during Lobectomy for Early-Stage Lung Cancer: An Analysis from the National Cancer Data Base" by Medbery et al. J Thorac Oncol 2016;11:e103-4. 